\title{
Small Plasma Focus Studied as a Source of Hard X-Ray
}

\author{
Fermín Castillo-Mejía, Maria Magdalena Milanese, Roberto Luis Moroso, Jorge Osvaldo Pouzo, and \\ Martin A. Santiago
}

\begin{abstract}
An experimental study on hard X-ray pulses emitted by a small plasma focus is performed, regarding its application to biological radiographs in fast dynamic situations. It is found that the radiation intensity in a single shot is high enough to obtain fine resolution radiographs in very short exposures (about $10 \mathrm{~ns}$ ). As an example, a radiograph on a live mouse is shown. It is found that the relation between responses of different detectors, i.e., thermoluminiscence dosimeters and scintillator-photomultiplier, is linear.
\end{abstract}

Index Terms-Plasma focus, radiography, X-ray sources.

\section{INTRODUCTION}

$\mathbf{T}$ HE DENSE plasma focus device (DPF) [1]-[5] produces a hot dense plasma by the rapid magnetic compression of a plasma cylinder formed at the end of a coaxial electrode system (see Fig. 1). When pure deuterium is used as filling gas, fusion reactions $\left(D+D \rightarrow \mathrm{He}^{3}+n\right)$ are produced in about $100 \mathrm{~ns}$ of hot plasma duration. These reactions could be produced by two mechanisms: 1) thermonuclear mechanism, present in the plasma bulk, where the reactions are produced by thermal collisions of deuterons; and 2) beam-target mechanism, due to selective acceleration of deuterons (and electrons in opposite direction) in strong electric fields originated in $m=0$ instabilities along the plasma column. The thermonuclear mechanism produces a soft X-ray emission (of some $\mathrm{keV}$ ) [6]-[8] originated by bremsstrahlung of electrons on deuterons of the thermal plasma. $\mathrm{X}$-ray pulses could also be originated because the interaction between the current sheath and the anode [9]. The nonthermal mechanism produces a high-energy ion beam and, in opposite direction, an electron beam that collides with the anode surface originating a short pulse of hard X-ray emission [6]. Recent works [10], [11], have shown the coexistence of both mechanisms and the respective influence in the nuclear reaction production. The main scope of this paper is the study of the hard $\mathrm{X}$-ray emission (mechanism $\mathrm{b}$ ) in order to have information on the peak duration, source size and radiation intensity. All this information is driven, in this study, to the application of special radiography of dynamic biological systems, where high spatial

Manuscript received April 16, 2001; revised June 22, 2001. This work was supported in part by the Center of Buenos Aires Province University, the National Research Council, and the Buenos Aires Regional Research Council.

F. Castillo-Mejía is with the Institute of Nuclear Sciences, National Autonomous University of Mexico, Mexico City 04510, Mexico.

M. M. Milanese, R. L. Moroso, and J. O. Pouzo are with the Institute of Physics Arroyo Seco, Faculty of Sciences, Center of Buenos Aires Province University, Tandil, Argentina and are also with the National Research Council, Tandil, Argentina (e-mail: pouzo@exa.unicen.edu.ar).

M. A. Santiago is with the Institute of Physics Arroyo Seco, Faculty of Sciences, Center of Buenos Aires Province University, Tandil, Argentina.

Publisher Item Identifier S 0093-3813(01)11203-8.

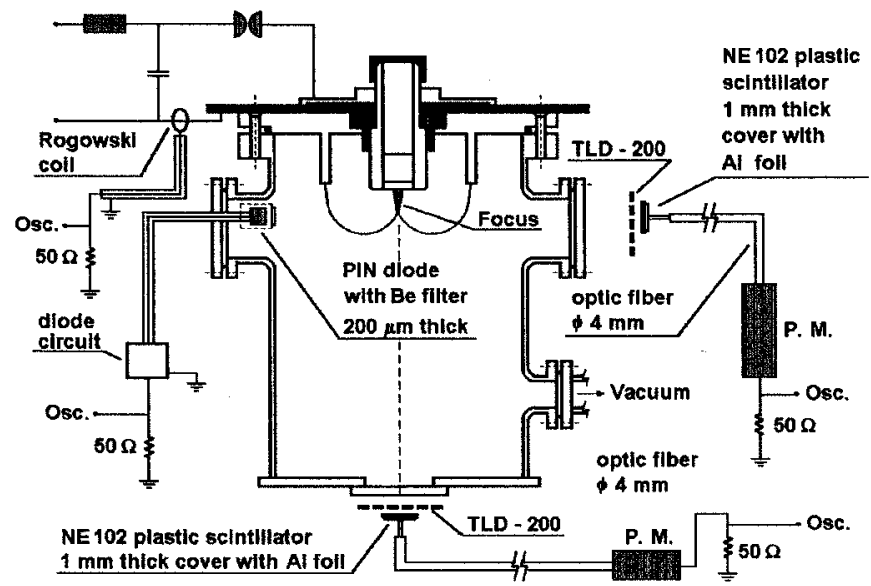

Fig. 1. Experimental setup for NE-102 scintillator-photomultiplier and TLD-200 chips for $0^{\circ}$ and $90^{\circ}$, including a PIN diode soft X-ray detector.

resolution (microscopy resolution) and short (but intense) time exposures are desired.

In this work, the soft X-ray and neutron emissions are checked simultaneously with the hard X-rays in order to have supplementary information on the plasma parameters. That is, these measurements are performed as collateral diagnostics for the generated plasma. It must be mentioned that, if only hard $\mathrm{X}$-ray application is the final scope, different filling gases can be used. In this work, we used deuterium in order to have the neutron measurement as plasma diagnostics.

\section{EXPERIMENTAL METHOD}

The DPF used is PACO [12], the coaxial electrodes (4 and 11 $\mathrm{cm}$ in diameter for the inner and outer electrode, respectively) are made in brass; the energy in the bank is $2 \mathrm{~kJ}$ when it is operated at $31 \mathrm{kV}$. At this voltage, the maximum discharge current $I_{m}$ reaches $250 \mathrm{kA}$ and, using $D_{2}$ at $1.5 \mathrm{mb}$ as filling gas, the fusion neutron yield $Y$ results in average $\sim 2 \times 10^{8}$ per shot in $4 \pi$ sr. The value $Y$ was measured, in each shot, with a silver activation counter detector. In Fig. 1, a scheme of the DPF system and the experimental arrangement are shown.

The hard X-ray radiation was detected simultaneously by means of two different systems: scintillator-photomultiplier (SPM) detector and thermoluminescence dosimeters (TLD). These two diagnostics allow, respectively, time-resolved and time-integrated measurements of X-ray pulses. The scintillator NE-102 used in the SPM measurement is very thin $(0.5-\mathrm{mm}$ thick), in order to minimize the detection of the $D-D$ fusion neutrons. It was covered with an $\mathrm{Al}$ foil to shield of the visible 


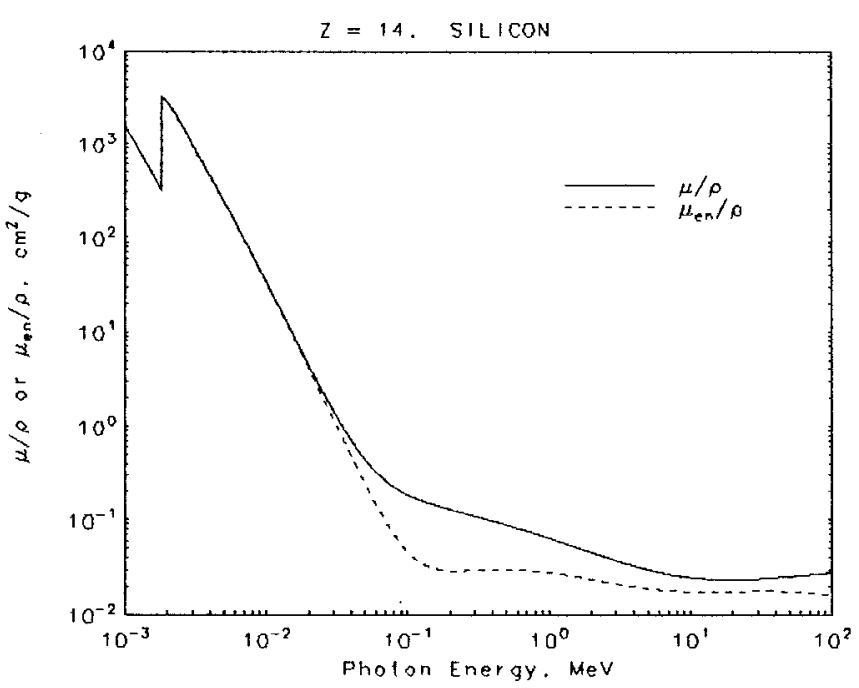

(a)

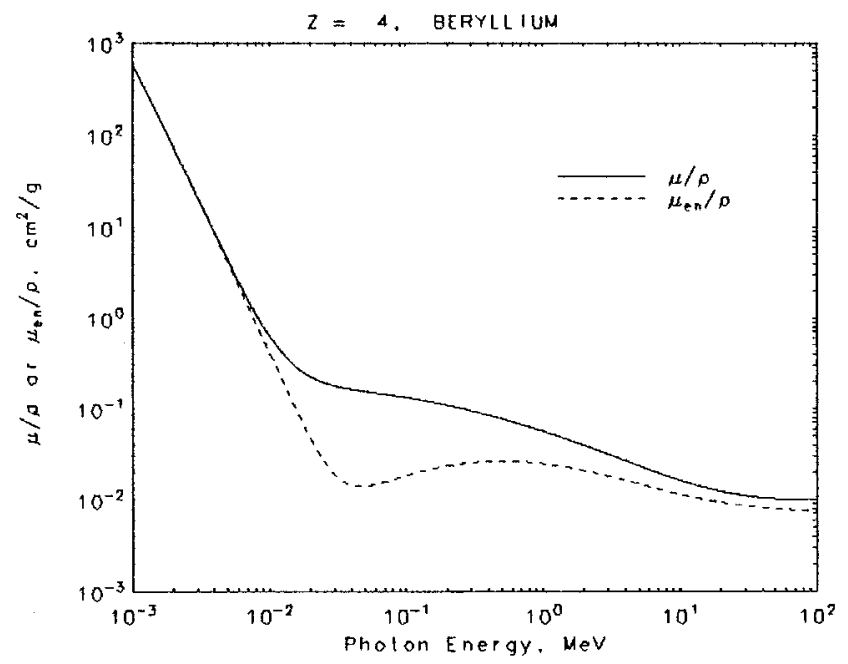

(b)

Fig. 2. Curve of the mass attenuation coefficient $\mu / \rho$ (continuous curve) as a function of photon energy for: (a) glass, and (b) beryllium.

light, and connected with the photomultiplier through an optical fiber. Both SPM and TLD systems are located outside the discharge chamber in front of a 5-mm thick glass window (see Fig. 1). This window constitutes a filter for X-rays with energies below $25 \mathrm{keV}$ [13]; then this is the lower energy threshold in our hard X-ray measurements. In Fig. 2(a), the curve of the mass attenuation coefficient (continuous curve) as a function of photon energy, for glass is given. Simultaneously, time-resolved soft X-ray pulse emission was registered using a fast positive-intrinsic-negative (PIN)-diode located into the chamber and shielded for visible light with a Be window (200- $\mu \mathrm{m}$ thick). In Fig. 2(b), the curve of the mass attenuation coefficient (continuous curve) as a function of photon energy, for Beryllium is given. Then, the PIN detector results sensitive in the energy range from 1 to $20 \mathrm{keV}$ (see [14]). In addition, the DPF discharge current was registered with a calibrated Rogowski coil. The three electrical signals were registered in a digital oscilloscope, using compensateddelay cables in order to achieve correct comparisons in timeresolved measurements.
As mentioned above, the time-integrated X-ray intensity was measured by using TLD chips. The thermoluminescence (TL) technique relies on the experimental fact that a previously irradiated dielectric and semiconductor material emits light if it is heated [15]. This property can be simply explained in the framework of the energy-band model by assuming that defects breaking translation symmetry can bring about metastable states (traps) inside the bandgap. Some of these traps are filled with charge carriers released during irradiation and, depending on their mean-life times, they can remain occupied for years at room temperature. When the temperature is increased, thermal stimulation supplies the trapped charges enough energy to relax to a ground state. TL is observed whenever the relaxation is accompanied by the release of the stored energy as photons. The usual TL experiment consists in measuring the intensity of the light emitted by the irradiated sample as long as its temperature is linearly increased. The obtained light intensity versus temperature curve is dubbed glow curve and usually shows several peaks, each of them corresponding to a different kind of trap (see Fig. 4). Since the light yield is proportional to the imparted dose in most of TL materials, investigations have been carried out in order to develop materials suitable to be used as radiation dosimeters [16]. In fact, nowadays there exist several commercial phosphors, available either in powder or in chip form, which are routinely used in radiotherapy and nuclear facilities. Other applications of the TL technique can be mentioned, such as dating of geological and archaeological samples, environment monitoring, and basic research of defects in solids.

TL detectors usually show a linear dose response in a wide range of photon energies ranging from $\mathrm{keV}$ to $\mathrm{MeV}$, and can be easily calibrated by means of standard radionuclide sources in order to obtain an absolute measurement of the dose absorbed by the dosimeters. Detection of X-ray radiation by means of TLD is perturbed neither by electrical interference nor by strong magnetic fields. By placing many TLD in different locations of the experimental setup, a space-resolved dose map can be obtained. Furthermore, several sets of TLD can be irradiated in different conditions and be measured later. To the best of our knowledge, the first use of thermoluminescent dosimeters $\mathrm{CaF}_{2}$ : Dy (TLD-200) and LiF:Mg Ti (TLD-100) as X-ray detectors for plasma diagnostics was reported by Gullickson et al. [17]. More recently, an application of TLD to absolute brightness measurements of nanosecond X-ray pulses has been reported [18]. Commercially available dysprosium-doped calcium fluoride $\left(\mathrm{CaF}_{2}\right.$ : Dy) TLD-200, $3.2 \times$ $3.2 \times 0.9-\mathrm{mm}$ chips (Bicron-NE/Harshaw, USA), was used at the present study. For the purpose of X-ray diagnostics, the dosimeters were carefully sorted with respect to their sensitivity to ensure a $5 \%$ standard precision of the calibration. The energy calibration was performed by means of a ${ }^{60} \mathrm{Co}$ standard radioactive source $(5000 \mathrm{Ci})$ in the 5 to $250 \mathrm{rad}$ range. An annealing treatment at $400{ }^{\circ} \mathrm{C}$ for two hours followed by another annealing at $100{ }^{\circ} \mathrm{C}$ for two hours, were carried out on the TLD before each irradiation. Prior to irradiation, the TL chips were covered with a $0.1-\mathrm{mm}$ thick $\mathrm{Al}$ film to prevent light produced TL fading. The glow curves were measured by using a cooled photomultiplier tube EMI 6097B and a PC-aided Harshaw 2000-B picoamperimeter, and with a heating rate of 


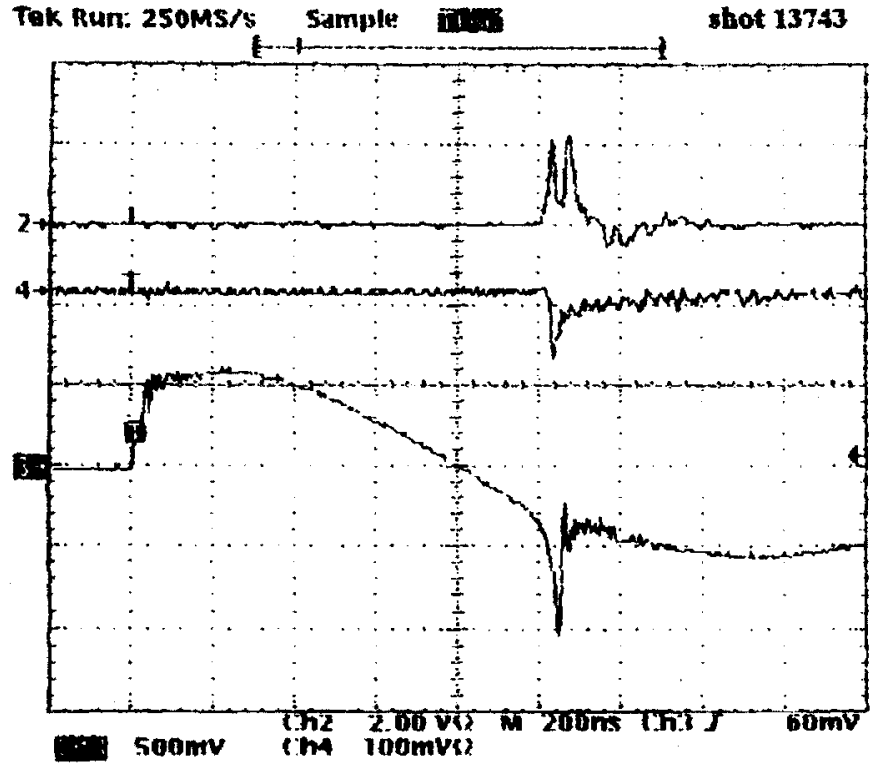

Fig. 3. Typical waveforms from PIN diode, scintillator-PM detector with a thin Al filter and Rogowski coil in top, middle, and bottom traces, respectively.

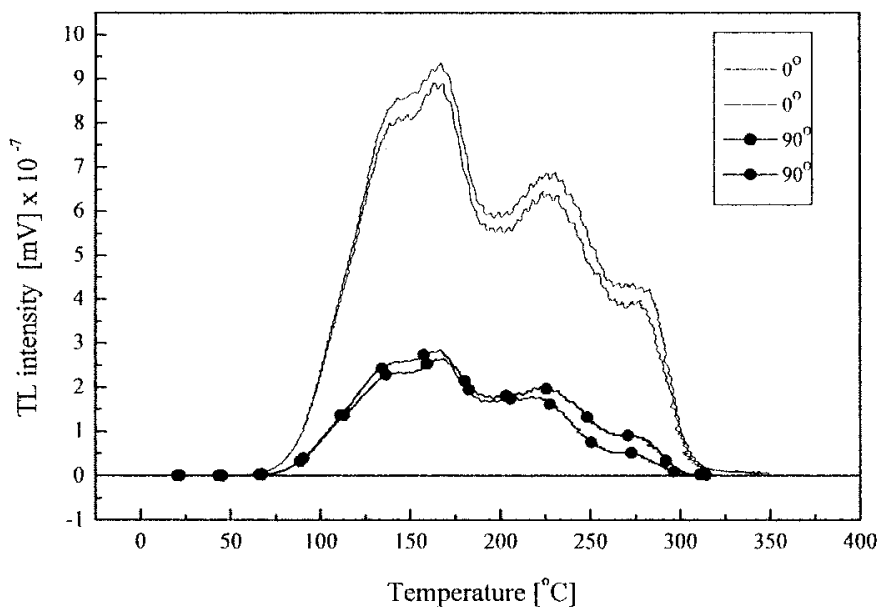

Fig. 4. Glow curves of TLD-200 chips irradiated with hard X-ray from DPF at two different angles.

$3{ }^{\circ} \mathrm{C} / \mathrm{s}$ between $50{ }^{\circ} \mathrm{C}$ and $350{ }^{\circ} \mathrm{C}$. The dose absorbed by the dosimeters has been estimated by means of the peak II height ( $165^{\circ} \mathrm{C}$ at this heating rate), and the lapse between irradiation and reading was always of one day.

Spatial distribution (assuming a cylindrical symmetry) of $\mathrm{X}$-ray in each DPF shot was determined locating a set of TLD chips at $1 \mathrm{~m}$ in front of the anode end (outside the chamber) by placing them every $6^{\circ}$ for $0^{\circ} \leq \theta<30^{\circ}$ (see Fig. 4). Three detectors were recorded for each angle, in order to check some difference in the response of the detectors. Anyway, all the used TLD chips were previously checked (during their calibration), and the difference of response between chips is less than $5 \%$.

\section{RESUlTS AND COMMENTS}

\section{A. Correlation Between TLD and SPM Detection}

Response at TLD-200 (in dose) was compared with the maximum amplitude (in volts) at SPM signal peak. Typical oscillo-

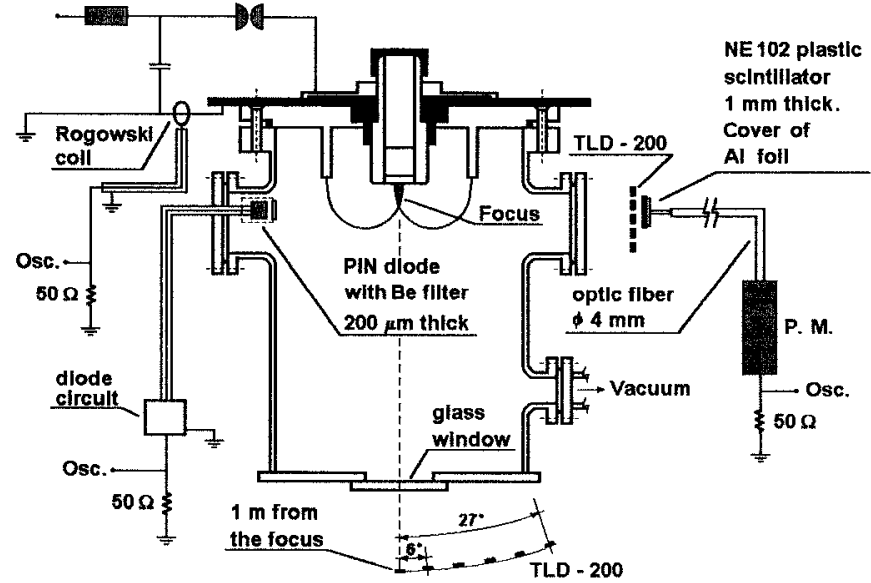

Fig. 5. Experimental setup of the TLD in different angular positions respect to the electrodes axes.

grams obtained in the digital Tektronix TDS 540A are shown in Fig. 3, with PIN diode, SPM, and Rogowski coil signals in top, middle, and bottom traces, respectively. We obtain the timing of SPM and PIN diode signals with respect to the minimum of the $d I / d t$ dip. The time correlation between signals is measured with an error less than 2 ns. In Fig. 4, it is shown typical glow curves registered to $0^{\circ}$ and $90^{\circ}$-direction respect to DPF axes. For correlating the TLD response versus scintillator-PM signal, both of them were placed at $0^{\circ}$ and $90^{\circ}$ outside the discharge chamber in front of a 5-mm thick glass window. Then, their responses were measured in individual DPF shots. The comparative response graphics for $0^{\circ}$ and $90^{\circ}$ are shown in Fig. 6(a) and (b), respectively. In these graphs, the correlation coefficient of linear relation is better than 0.9 .

Simultaneous measurements with TLD chips located at different distances to the focus, confirm that the dose depends on the inverse to the square of the source-detector distance.

Quantitatively, the dose measured at $1 \mathrm{~m}$ in front of the focus, results of about $0.1 \mathrm{mrad}$ per shot. This level of radiation is of the same order of magnitude that this of typical medical radiographs.

\section{B. Spatial Distribution on Hard X-Ray Radiation}

Being the hard $\mathrm{X}$-ray radiation is more intense at $\theta=0$, the dose was measured (in details) in this direction. Fig. 5shows the TLD chips setup. In the graphic of Fig. 7, it is shown the measured X-ray dose as a function of the angle with the TLD chips setup shown in Fig. 5, the measurements X-ray dose as a function of angle $\theta$. The window of observation is covered by an iron disk 10-mm thick, which stops the X-rays with energies under $100 \mathrm{keV}$ [13]. The pattern corresponds to the radiation through the smaller glass window, and the dose presents a plateau. Therefore, the dose results practically constant into the window aperture (at $1 \mathrm{~m}$ from the anode surface). Similar result is obtained when the chip set is located (at the same distance) on a plane surface.

\section{Image of Biological Specimens}

The plasma focus is an intense pulsed X-ray source. It delivers radiation enough, in a single shot of nanosecond duration, 


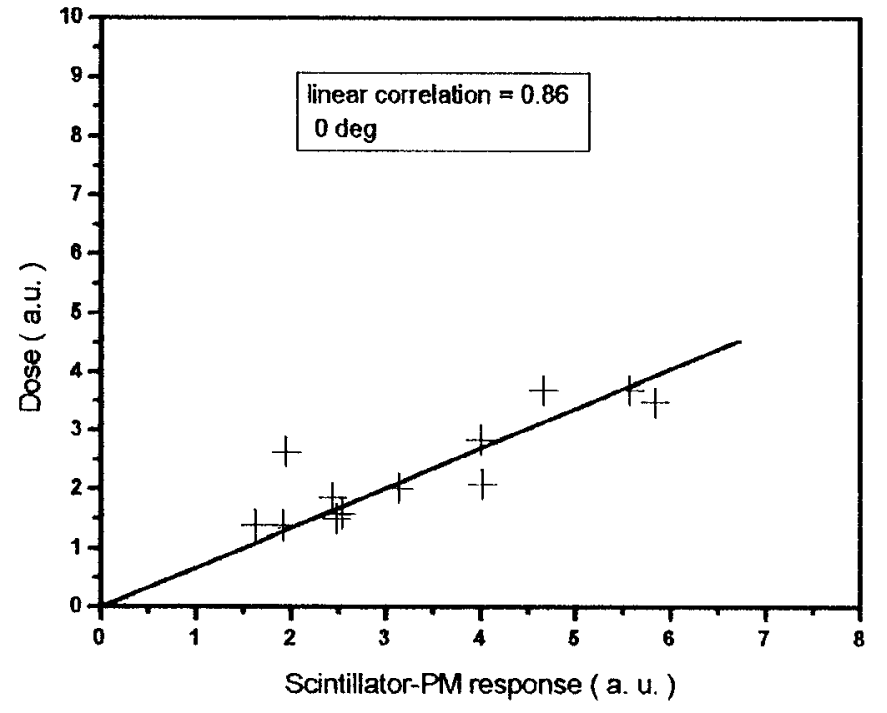

(a)

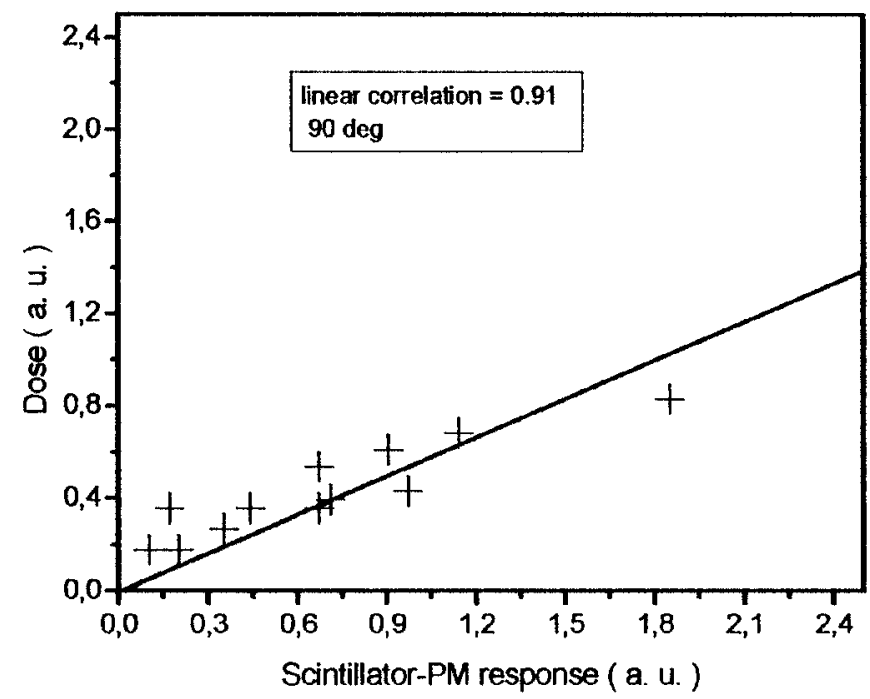

(b)

Fig. 6. Scintillator-PM detector signal versus TLD response for X-rays from a DPF; (a) detectors placed at $0^{\circ}$; (b) at $90^{\circ}$ respect to the electrode axis. Error bars are of order of $10 \%$.

to produce ultra fast radiography of biological specimens. It is reported here the preliminary results of the use of a low energy DPF device as intense pulsed X-ray source to produce an image of alive small biological objects (shown in Fig. 8). That figure shows an X-ray image of a mouse put at one meter from the focus $\left(\theta=0^{\circ}\right)$ with a single shot. The fine structure of the specimen can be seen in different parts. Such resolution corresponds to a source with a size approximately $0.1 \mathrm{~mm}$ or less. The limit of the picture corresponds to the projection of the glass window, and the white circle behind the mouse corresponds to a $1-\mathrm{cm}$ high iron cylinder that was used for the test.

\section{CONCLUSION}

The linearity between SPM amplitude peak and the dose measured with TLD, offers a special advantage. In a radiography exposure plate, it is possible to know (immediately) if the dose was enough to afford a sharp picture without previous development.

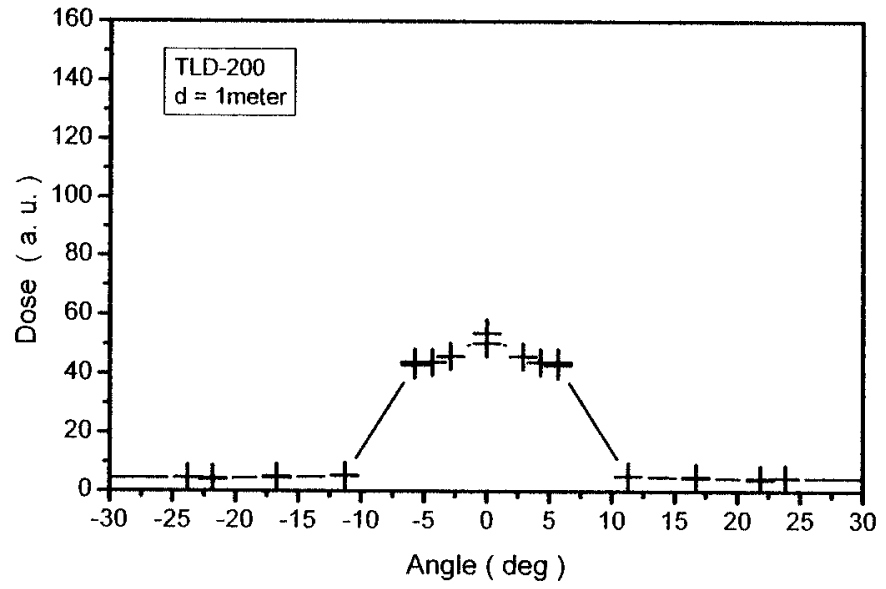

Fig. 7. X-ray intensity detected with TLD as function of angle according to the experimental arrangement in Fig. 5.

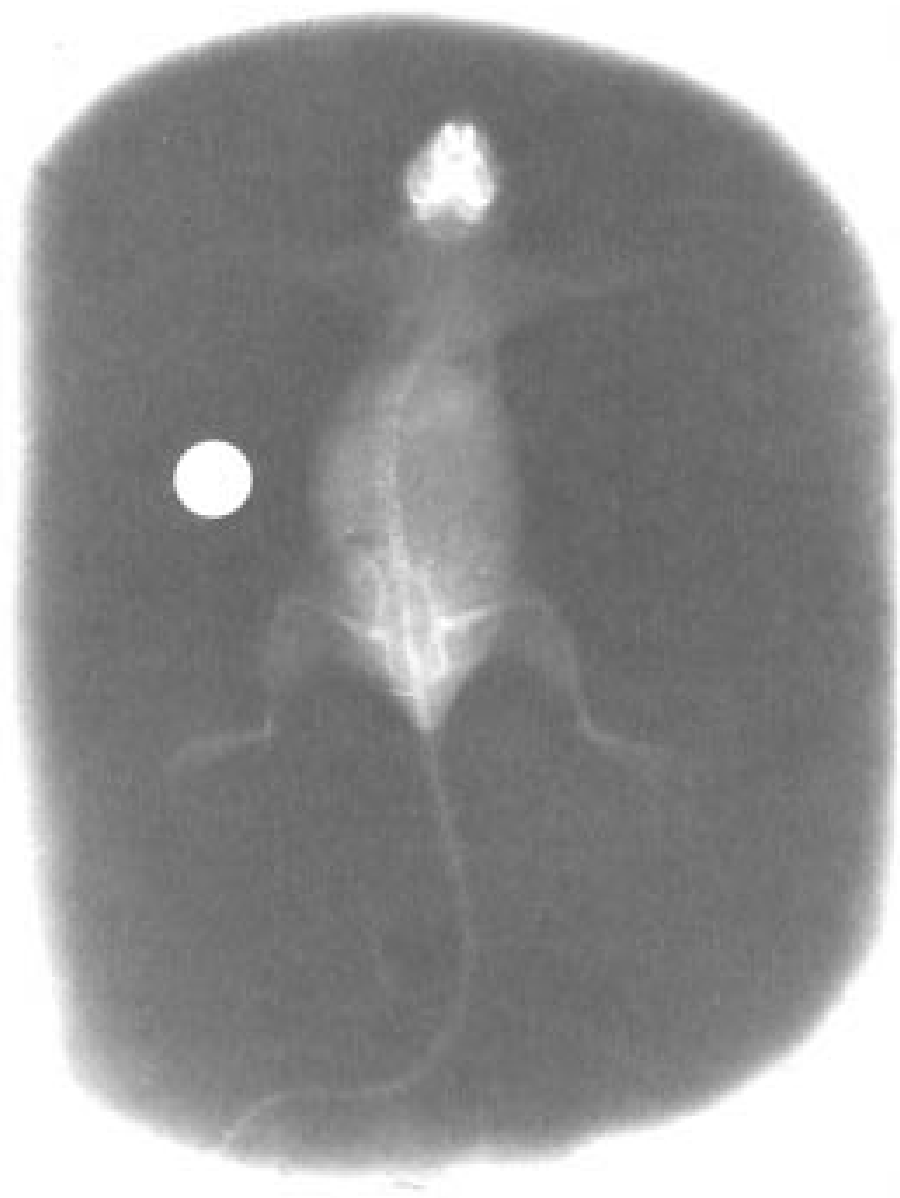

Fig. 8. X-ray image of a mouse taken with a single shot of the DPF at one meter to focus, the detailed structure of the specimen can be seen.

The uniform emission and level of doses that can be obtained in a relatively large zone at $1 \mathrm{~m}$ in front of the focus allow to obtain requested quality illumination (planar wave) for radiographs.

The high spatial resolution of the radiography, which corresponds to a source with a typical size in the order of $0.01 \mathrm{~mm}$ (diameter), is one of the most relevant results into the interest of 
the application in biological radiography. Moreover, this result opens the possibility to use this system in microradiographies.

From the point of view of DPF phenomenon research, the fact that the source is so small reaffirms the hypothesis that the hard $\mathrm{X}$-ray pulses could be generated by collimated electron beams incident on the anode surface.

The main advantage of this system is the very short time of X-ray pulse (about $20 \mathrm{~ns}$ ), which allows researchers to obtain high-quality radiographies of very dynamic biological systems (e.g., blood in circulation).

It must also be mentioned the fact that the dose can be varied, not only with the distance, but also using different filling gases or changing the anode material. With such changes, the dose can be varied by one order of magnitude. Changes in the operating voltage of the dense plasma focus can also be a method to gradually vary the dose.

\section{ACKNOWLEDGMENT}

The authors with to thank Dr. R. Najle and his assistant Med. I. Betelú for their help in the biological radiography experiment. They also thank the authorities of Nuclear Science Institute, UNAM, Mexico, for the support to their collaboration program.

\section{REFERENCES}

[1] J. W. Mather, Dense Plasma Focus Methods of Experimental Physics, R. Lovberg and H. Griew, Eds. New York: Academic, 1971, vol. 9B, p. 187.

[2] V. Imshennik, N. Filippov, and T. Filippova, "Similarity theory and increased neutron yield in plasma focus," Nucl. Fusion, vol. 30, p. 929, 1973.

[3] G. Decker and R. Wienecke, "Plasma Focus Devices," Physica, vol. 82c, p. $155,1976$.

[4] M. Sadowski, H. Herold, H. Schmidt, and M. Shakhatre, "Filamentary structure of the pinch in plasma focus discharges," Phys. Lett., vol. 105a, pp. 117-123, 1984.

[5] S. Lee, "Technology of small plasma focus incorporating some experiences with the UNU/ICTP PFF," Small Plasma Phys. Exp. II, pp. $113-169,1990$.

[6] M. Zakaullah, I. Akhtar, A. Waheed, K. Alamgir, A. Z. Shah, and G. Murtaza, "Comparative study of ion, X-ray and neutron emission in a low energy plasma focus," Plasma Sources, Sci. Technol., vol. 7, pp. 206-218, 1998.

[7] A. Serban and S. Lee, "Soft X-ray emission from small plasma focus operated in deuterium," Plasma Sources Sci. Technol., vol. 6, pp. 78-85, 1997.

[8] P. Choi and M. Favre, "Silver as a soft X-ray filter for plasma diagnostics," J. Phys. D, Appl. Phys., vol. 20, pp. 169-173, 1987.

[9] M. Zakaullah, K. Alamgir, M. Shafiq, S.-M. Hassan, and M. Shariff, "Enhanced copper K-alpha radiation from a low-energy plasma focus," Appl. Phys. Lett., vol. 78, pp. 877-879, 2001.

[10] F. Castillo-Mejía, M. Milanese, R. Moroso, and J. Pouzo, "Experimental researches of X-ray and neutron emission in a dense plasma focus," in Proc. VIII Latin-American Workshop on Plasma Physics (LAWPP'98), vol. I, Contributed Papers, Tandil, Argentina, Nov. 16-27, 1998, pp. $\mathrm{V}-2.1-\mathrm{V}-2.8$.

[11] _ _Evidences of thermal and nonthermal mechanisms coexisting in a dense plasma focus D-D nuclear reactions," J. Phys. D, Appl. Phys., vol. 33, pp. 141-147, 2000.

[12] M. Milanese, R. Moroso, and J. Pouzo, "Dynamics of the ionizing and magnetic fronts in the radial compression stage of DPF current sheath," IEEE Trans. Plasma Sci., vol. 21, pp. 373-377, Aug. 1993.
[13] J. H. Hubbell and S. M. Seltzer. "Tables of X-ray mass attenuation coefficients and mass energy-absorption coefficients from $1 \mathrm{keV}$ to $20 \mathrm{MeV}$ for elements $Z=1$ to 92 and 48 additional substances of dosimetric interest". Ionizing Radiation Division, Physics Laboratory National Institute of Standards and Technology, Gaithersburg, MD 20 899. [Online]. Available: http://physics.nist.gov/PhysRefData/XrayMassCoef.

[14] C. S. Wong, "Five channel diode X-ray spectrometer," Instruction Manual, Univ. of Malaya, Malaysia, ICAC-UM/DXS-4, 1992.

[15] S. W. S. McKeever, M. Moskovitch, and P. D. Towsend, Thermoluminescence Dosimetry Materials: Properties and Uses. Ashford, U.K. Nuclear Technology , 1995.

[16] J. Azorín, C. Furetta, and A. Scacco, "Preparation and properties of thermoluminescent materials," Phys. Stat. Sol. (a), vol. 138, pp. 9-46, 1993.

[17] R. Gullickson and R. Barlett, "X-Ray analysis for electron beam enhancement in the plasma focus device," Advan. X-Ray Anal., vol. 18, pp. 184-195, 1974

[18] M. Fárníková, J. Krása, L. Juha, and L. Ryć, "Thermoluminescent dosimetry of X-ray radiation emitted from a laser-produced plasma," $J$. Phys. D, Appl. Phys., vol. 29, pp. 2119-2123, 1996.

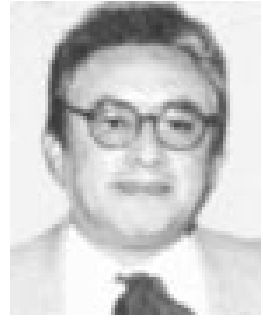

optic sounding.
Fermín Castillo-Mejía received the M.Sc. degree in physics from the National Autonomous University of Mexico (UNAM), and the Ph.D. degree in physics from the Center of Buenos Aires Province University (UNICEN), Tandil, Argentina, in 1990 and 1998, respectively.

$\mathrm{He}$ was previously an Invited Researcher at UNICEN. He is currently working at the Nuclear Sciences Institute, UNAM. His research interests include electrical discharges and several dense plasma focus diagnostics about neutron, X-ray, and

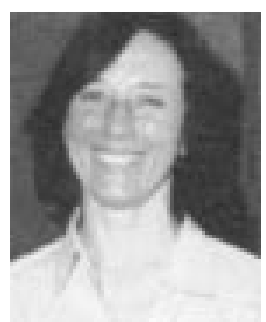

Maria Magdalena Milanese received the M.Sc. degree in physics and the Ph.D. degree in physics from Buenos Aires University (UBA), Argentina, in 1975 and 1988 , respectively.

She has had long research stays at the EURATOM 1 MJ PF, Frascati, Italy, the Electrotechnical Institute Galileo Ferraris, Torino, Italy, and in The Düsseldorf University, Germany, working in the SPEED 1 PF. Currently, she is a Professor with the Center of Buenos Aires Province University (UNICEN), Tandil, Argentina. Until 1982, she was with the Plasma Physics Laboratory of the UBA. Since 1983, she has been with the Plasma Group, Physics Institute Arroyo Seco, UNICEN. Since 1985, she has been with the Researcher Career of National Council of Scientific and Technological Researches, Argentina. She participated in numerous national and international symposia, and has co-authored about 60 published papers. Her research interests include experimental investigations in plasma focus $(\mathrm{PF})$, including neutron, charged particles, and electromagnetic studies, and PF criteria of design.

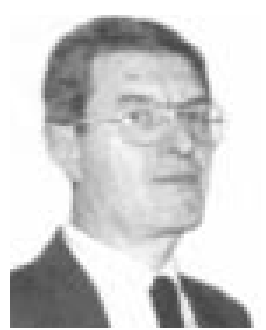

Roberto Luis Moroso received the M.Sc. degree in physics from the Center of Buenos Aires Province University (UNICEN), Tandil, Argentina, in 1971.

From 1972 to 1983 , he was a Lecturer with the Physics Department, UNICEN. He joined the Plasma Physics Group in 1984. From 1987 to 1991, he shared the time with the Plasma Lab, LaFyP, Mar del Plata University. His research interests include electrical discharges and several dense plasma focus diagnostics about gas puff and gas jet operation. 


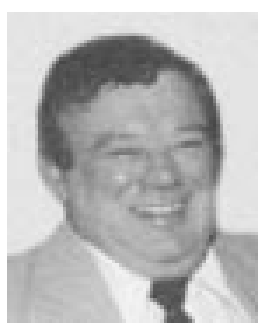

Jorge Osvaldo Pouzo obtained his Master in physics degree in 1973 and his Ph.D. in physics in 1979 in Buenos Aires University, Buenos Aires, Argentina.

He had research stays in several Centers of America and Europe, including the EURATOM 1 MJ Plasma Focus, Frascati, Italy, the Electrotechnical Institute Galileo Ferraris, Torino, Italy, Düsseldorf University, Germany (as A. Humboldt Fundation fellow), National Autonomous University of Mexico, Mexico City, Mexico, and Soltan Institute for Nuclear Studies, Warsaw, Poland. Until 1982, he was with the Plasma Physics Laboratory, Buenos Aires University, Argentina. Since 1983, he has been with the Research Career of National Council of Scientific and Technological Researches, Argentina, and he has been the Director of the Plasma Group, Physics Institute Arroyo Seco, Center of Buenos Aires Province University (UNICEN), Tandil, Argentina, and the Laboratory of Fluid-dynamics and Plasma, Mar del Plata University (UNMdP), Mar del Plata. He is currently a full Professor at UNICEN, and a part-time Professor at UNMdP. He has participated in both national and international symposia, and is the author of numerous published papers and several invited papers in international conferences. His research interests include plasma focus investigations such as neutron, charged particles and electromagnetic studies, and plasma focus criteria of design.

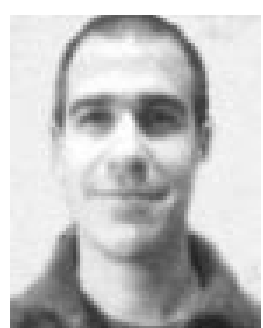

Martin A. Santiago received the M.Sc. degree in physics from the Center of Buenos Aires Province University, Tandil, Argentina, in 1998, and currently holds a fellowship of the National Council of Scientific and Technological Researches at the same university.

His research interests include luminescence of solids, thermoluminescence dosimetry, radiation-induced defects in borates, and digital mammography processing. 